\title{
PRODUÇÃO DE BIOMASSA EM PLANTAS DE $P$. angulata L. SOB DIFERENTES DOSES DE MACRONUTRIENTES
}

\author{
Viviane de Jesus Carneiro'; Marilza Neves do Nascimento Ribeiro² ${ }^{2}$ : Alismário Leite da \\ Silva $^{3}$ e Francisco dos Santos Neto ${ }^{4}$ \\ 1. Bolsista PIBIC/FAPESB, Graduanda em Agronomia, Universidade Estadual de Feira de Santana, e-mail: \\ vivianedjcarneiro@gmail.com \\ 2. Orientadora, Departamento de Ciências Biológicas, Universidade Estadual de Feira de Santana, e-mail: \\ marilzaagro@hotmail.com \\ 3. Coorientador, Mestrando em Recursos Genéticos Vegetais, Universidade Estadual de Feira de Santana, e-mail: \\ alismarioagronomo@hotmail.com \\ 4. Graduando em Agronomia, Universidade Estadual de Feira de Santana, e-mail: franciscosantosn@ @hotmail.com
}

PALAVRAS-CHAVE: Physalis; nutrição; matriz baconiana.

\section{INTRODUÇÃO}

A Physalis angulata L., também conhecida por camapú ou joá-de-capote, é encontrada em diversas regiões do Brasil, sendo considerada planta invasora quando próxima a outras culturas (Corrêa, 1984). Por expressar grande potencial farmacológico, por conta da produção de fisalinas (Adams et al., 2009), muitos estudos têm sido realizados sobre a espécie, entretanto, sabe-se pouco sobre o seu manejo nutricional, mesmo sendo um importante fator dentro de um cultivo.

Dos elementos essenciais, seis são requeridos em maiores quantidades, que são os macronutrientes, sendo eles: nitrogênio $(\mathrm{N})$, fósforo $(\mathrm{P})$, potássio $(\mathrm{K})$, cálcio $(\mathrm{Ca})$, magnésio $(\mathrm{Mg})$ e enxofre $(\mathrm{S})$ e, as quantidades de nutrientes exigidas para o correto desenvolvimento e obtenção de altas produções, variam de acordo com a espécie (SHEAR e FAUST, 1980).

Tendo em vista que a produção de fisalinas se dá principalmente nos órgãos da parte aérea (Simões, 1999), deve-se buscar uma recomendação para a $P$. angulata visando o aumento da produção de biomassa, pois com as quantidades adequadas de nutrientes, pode-se alcançar a máxima eficiência da espécie. Diante disso, o presente trabalho buscou avaliar o efeito dos macronutrientes na produção de biomassa em plantas de Physalis angulata L.

\section{METODOLOGIA}

Conduzido na Unidade Experimental Horto Florestal da Universidade Estadual de Feira de Santana, BA, os tratamentos foram obtidos segundo o arranjo estatístico matriz Baconiana (Turrent, 1979), em delineamento inteiramente casualizado, sendo avaliados os nutrientes: $\mathrm{N}, \mathrm{P}, \mathrm{K}, \mathrm{Ca}, \mathrm{Mg}$ e $\mathrm{S}$ em três diferentes doses, sendo estas, N: 75, 225 e 300mg.dm ${ }^{-3}$, P: 30, 90 e $120 \mathrm{mg}^{-\mathrm{dm}^{-3}}$, K: 20, 60 e $80 \mathrm{mg} \cdot \mathrm{dm}^{-3}$, Ca: 0,5, 1,5 e 2,0 $\mathrm{cmol}_{\mathrm{c}} \mathrm{dm}^{-3}, \mathrm{Mg}: 0,2,0,6$ e 0,8 $\mathrm{cmol}_{\mathrm{c} .} \mathrm{dm}^{-3}$. Haviam também dois tratamentos adicionais, o de referência e o controle, sem a adição de nutrientes, com cinco repetições, totalizando 20 tratamentos. A dose de referência foi estabelecida a partir da recomendação para a cultura do tomate: $\mathrm{N} 150 \mathrm{mg} . \mathrm{dm}^{-3}, \mathrm{P} 60 \mathrm{mg} \cdot \mathrm{dm}^{-3}, \mathrm{~K} 150 \mathrm{mg} . \mathrm{dm}^{-3}$, Ca

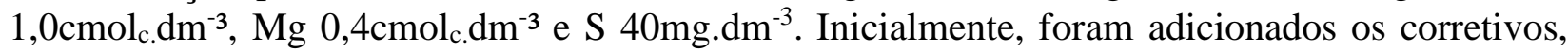
$\mathrm{CaCO}_{3}$ e $\mathrm{MgCl}$. Passados 50 dias, os demais nutrientes foram adicionados. Ao final do experimento as plantas coletadas, foram submetidas às análises de área foliar (AF), massa seca total (MST) e massa da parte aérea (MSPA). Os dados obtidos, foram interpretados estatisticamente por meio de análises de regressão, utilizando-se o programa Estatístico SISVAR® 5.3 (Ferreira, 2008), a 1 e 5 $\%$ de probabilidade.

\section{RESULTADOS E DISCUSSÃO}

\section{Efeito do Nitrogênio}

A aplicação de nitrogênio mostrou-se significativa para todos os parâmetros analisados. O modelo de regressão que melhor se ajustou aos dados foi de ordem quadrática (Tabela 1). A dose estimada para a AF promoveu aumento de $258 \%$ nas respostas do parâmetro, quando comparadas 
ao tratamento controle. Já para a MST o incremento promovido pela dose estimada foi 315,4\%, em relação ao tratamento controle e, para a MSPA promoveu incremento de 203,1\% em relação ao mesmo tratamento.

Tabela 1. Resumo das estimativas geradas do efeito da aplicação de doses crescentes de Nitrogênio em mg.dm ${ }^{-3}$, sobre as seguintes características: área foliar (AF), massa seca total (MST) e massa seca da parte aérea (MSPA).

\begin{tabular}{cccc}
\hline Parâmetro & Equação & $\mathrm{R}^{2}(\%)$ & Dose estimada $\left(\mathrm{mg}^{\mathrm{d}} \mathrm{dm}^{-3}\right)$ \\
\hline AF & $\mathrm{Y}^{* *}:-0,1004 \mathrm{x}^{2}+29,293 \mathrm{x}+936,01$ & 95.46 & 145,88 \\
MST & $\mathrm{Y}^{* *}:-0,0013 \mathrm{x}^{2}+0,3463 \mathrm{x}+6,1001$ & 94.68 & 133,19 \\
MSPA & $\mathrm{Y}^{* *}:-0,0005 \mathrm{x}^{2}+0,1276 \mathrm{x}+4,6211$ & 92.94 & 127,60 \\
\hline
\end{tabular}

**: Significativo a $1 \%$ de probabilidade; *: Significativo a $5 \%$ de probabilidade.

Este incremento promovido pelo nutriente nestes parâmetros, pode estar relacionado ao aumento da área foliar das plantas, apontando o nitrogênio, como um fator limitante na produção vegetal (Yasari et al. 2009), além disso, o nutriente é responsável pelo aumento da conversão de carboidratos em proteínas, aumentando assim o tamanho das células do vegetal, promovendo o crescimento vegetativo (MARSCHNER, 1995).

\section{Efeito do Fósforo}

A aplicação de Fósforo influenciou significativamente os resultados para todos os parâmetros analisados, apresentando efeito de ordem quadrática (Tabela 2). A AF no tratamento controle foi $264,5 \%$ inferior, quando comparada às respostas promovidas pela dose estimada. Em relação a MST a dose estimada, proporcionou incremento de $20,65 \mathrm{~g}$, sob as respostas do tratamento controle, expressando um aumento de $394,16 \%$. Já em relação ao tratamento com a dose referência, esse aumento foi de $9,8 \%$. E, para a MSPA, com dose estimada de 77,54mg.dm ${ }^{-3}$ o acréscimo foi de $215,4 \%$ em relação ao tratamento controle.

Tabela 2. Resumo das estimativas geradas do efeito da aplicação de doses crescentes de Fósforo em mg.dm-3, sobre as seguintes características: área foliar (AF), massa seca total (MST) e massa seca da parte aérea (MSPA).

\begin{tabular}{cccc}
\hline Parâmetro & Equação & $\mathrm{R}^{2}(\%)$ & Dose estimada $\left(\mathrm{mg}^{2} \mathrm{dm}^{-3}\right)$ \\
\hline AF & $\mathrm{Y}^{* *}:-0,4285 \mathrm{x}^{2}+60,528 \mathrm{x}+991,41$ & 93.38 & 70,63 \\
MST & $\mathrm{Y}^{* *}:-0,0044 \mathrm{x}^{2}+0,5979 \mathrm{x}+7,3622$ & 84.24 & 67,94 \\
MSPA & $\mathrm{Y}^{* *}:-0,0014 \mathrm{x}^{2}+0,2171 \mathrm{x}+4,8913$ & 91.29 & 77,54 \\
\hline
\end{tabular}

**: Significativo a $1 \%$ de probabilidade; *: Significativo a $5 \%$ de probabilidade.

Tais resultados indicam que houve aumento na produção de biomassa quando o nutriente foi fornecido. De acordo com Marschner (1995), essa elevação na produção de fitomassa está ligada ao fato do nutriente atuar como regulador no processo fotossintético, no metabolismo e na redistribuição de assimilados nas folhas.

\section{Efeito do Potássio}

A aplicação de potássio influenciou significativamente os resultados para todos os parâmetros analisados, sendo que o modelo que melhor se ajustou aos dados foi de ordem quadrática (Tabela 3). O parâmetro AF, com dose estimada de 214,62 $\mathrm{mg} \cdot \mathrm{dm}^{-3}$, expressou incremento de $253 \%$ em relação ao tratamento controle e, $6,8 \%$ em relação ao tratamento com a dose referência. Em relação a MSPA, o parâmetro apresentou dose estimada superior a 150 $\mathrm{mg}^{-\mathrm{dm}^{-}}$ ${ }^{3}$, promovendo incremento de 7,9g sob a MSPA. Já para a MST, o acréscimo proporcionado pela dose estimada sob o tratamento controle foi de $18 \mathrm{~g}$.

Tabela 3. Resumo das estimativas geradas do efeito da aplicação de doses crescentes de Potássio em mg.dm ${ }^{-3}$, sobre as seguintes características: área foliar (AF), massa seca total (MST) e massa seca da parte aérea (MSPA).

\begin{tabular}{cccc}
\hline Parâmetro & Equação & $\mathrm{R}^{2}(\%)$ & Dose estimada $\left(\mathrm{mg}^{2} \mathrm{dm}^{-3}\right)$ \\
\hline AF & $\mathrm{Y}^{* *}:-0,0443 \mathrm{x}^{2}+19,015 \mathrm{x}+989,14$ & 94.15 & 214,62 \\
MST & $\mathrm{Y}^{* *}:-0,0007 \mathrm{x}^{2}+0,223 \mathrm{x}+7,2703$ & 73.95 & 159,29 \\
MSPA & $\mathrm{Y}^{* *}:-0,0002 \mathrm{x}^{2}+0,0773 \mathrm{x}+4,6953$ & 93.61 & 193,25 \\
\hline
\end{tabular}

**: Significativo a $1 \%$ de probabilidade; *: Significativo a $5 \%$ de probabilidade. 
Este aumento nos parâmetros de massa seca era esperado, já que houve acréscimo na área foliar, que pode ser explicado pela função do potássio no alongamento celular. Além disso, o nutriente, por ser um importante regulador da turgidez dos tecidos, influencia diretamente o armazenamento de água nos tecidos, atuando como um promotor de condições ideais para as reações fotossintéticas e outros processos metabólicos (MALAVOLTA, 1980).

\section{Efeito do Cálcio}

A aplicação de Cálcio influenciou significativamente os resultados para todos os parâmetros analisados. O modelo que melhor se ajustou aos dados dos parâmetros foi de ordem quadrática (Tabela 4). As doses estimadas dos parâmetros AF e MST, promoveram incremento de 253,2\%, $298,4 \%$ em relação ao tratamento controle. Para a MSPA a dose estimada proporcionou incremento de $12,80 \mathrm{~g}$ sob o parâmetro, quando comparado ao tratamento controle, e 4,84\% de acréscimo em relação ao tratamento com a dose referência.

Tabela 4. Resumo das estimativas geradas do efeito da aplicação de doses crescentes de Cálcio em $\mathrm{cmol}_{\mathrm{c}} \cdot \mathrm{dm}^{-3}$, sobre as seguintes características: área foliar (AF), massa seca total (MST) e massa seca da parte aérea (MSPA).

\begin{tabular}{cccc}
\hline Equâmetro & Equação & $\mathrm{R}^{2}(\%)$ & Dose estimada $\left(\mathrm{cmol}_{\mathrm{c}} \cdot \mathrm{dm}^{-5}\right)$ \\
\hline AF & $\mathrm{Y}^{* *}:-1295,1 \mathrm{x}^{2}+3208,3 \mathrm{x}+1044,8$ & 88.82 & 1,24 \\
MST & $\mathrm{Y}^{* *}:-14,69 \mathrm{x}^{2}+35,105 \mathrm{x}+6,9952$ & 86.25 & 1,19 \\
MSPA & $\mathrm{Y}^{* *}:-4,8479 \mathrm{x}^{2}+12,293 \mathrm{x}+5,0114$ & 86.67 & 1,27 \\
\hline
\end{tabular}

**: Significativo a $1 \%$ de probabilidade; *: Significativo a $5 \%$ de probabilidade.

Gomes et al. (2002), constataram que o fornecimento de doses crescentes de Cálcio, promoveu o aumento na produção de massa seca de plantas de alfafa. Tais efeitos podem ser explicados pela função desempenhada pelo cálcio, que está envolvido nos processos de alongamento e divisão celular (MALAVOLTA, 1980).

\section{Efeito do Magnésio}

A aplicação de Magnésio influenciou significativamente os resultados para todos os parâmetros analisados. As respostas destes parâmetros foram ajustadas ao modelo de regressão quadrática (Tabela 5). Os acréscimos promovidos pelas doses estimadas em relação ao tratamento controle foram para a AF, de 267,7\%, na MST de 291,2\% de aumento, MSPA com 323,5\% de aumento nas respostas do parâmetro em relação a este tratamento. $\mathrm{O}$ incremento promovido por estas doses foi de, respectivamente, 4,9g, 3,6g e 9,4 nas respostas destes parâmetros sob o tratamento controle.

Tabela 5. Resumo das estimativas geradas do efeito da aplicação de doses crescentes de Magnésio em $\mathrm{cmol}_{\mathrm{c}} \cdot \mathrm{dm}^{-}$

${ }^{3}$, sobre as seguintes características: área foliar (AF), massa seca total (MST) e massa seca da parte aérea (MSPA).

\begin{tabular}{cccc}
\hline Parâmetro & Equação & $\mathrm{R}^{2}(\%)$ & Dose estimada $\left(\mathrm{cmol}_{\mathrm{c}} \cdot \mathrm{dm}^{-3}\right)$ \\
\hline AF & $\mathrm{Y}^{* *}:-6137,2 \mathrm{x}^{2}+7308,8 \mathrm{x}+979,96$ & 96.04 & 0,59 \\
MST & $\mathrm{Y}^{* *}:-82,86 \mathrm{x}^{2}+81,924 \mathrm{x}+7,2095$ & 80.29 & 0,49 \\
MSPA & $\mathrm{Y}^{* *}:-22,267 \mathrm{x}^{2}+27,923 \mathrm{x}+4,8969$ & 92.65 & 0,63
\end{tabular}

**: Significativo a $1 \%$ de probabilidade; *: Significativo a $5 \%$ de probabilidade.

Golçalves et al. (2014), identificaram que a adição de magnésio apresentou efeito significativo na massa seca da parte aérea e massa seca total. De acordo com Kerbauy (2004), a deficiência em Magnésio afeta diretamente a taxa fotossintética, por conta da sua função na síntese de proteínas e, o seu excesso pode promover redução na produção, pois as plantas expostas à toxidez do nutriente têm suas folhas intensamente afetadas.

\section{Efeito do Enxofre}

A aplicação de enxofre influenciou significativamente os resultados para todos os parâmetros analisados, apresentando modelo de regressão quadrática, que melhor se ajustou aos dados (Tabela 6). A dose estimada para AF promoveu incremento de 259,7\% sob o parâmetro, 
comparado ao tratamento controle. Em relação a MST, a dose estimada foi responsável pelo aumento de 392,9\% do parâmetro. O parâmetro MSPA, apresentou acréscimo nas respostas de 9,3g, sob o tratamento controle. Os resultados demonstram que a ausência de enxofre no cultivo de $P$. angulata é limitante ao cultivo, pois os parâmetros do tratamento controle apresentaram respostas inferiores comparadas aos tratamentos com diferentes doses do nutriente.

Tabela 6. Resumo das estimativas geradas do efeito da aplicação de doses crescentes de Enxofre em mg.dm sobre as seguintes características: área foliar (AF), massa seca total (MST) e massa seca da parte aérea (MSPA).

\begin{tabular}{cccc}
\hline Parâmetro & Equação & $\mathrm{R}^{2}(\%)$ & Dose estimada $\left(\mathrm{mg}^{\mathrm{d}} \mathrm{dm}^{-3}\right)$ \\
\hline AF & $\mathrm{Y}^{* *}:-0,7865 \mathrm{x}^{2}+80,129 \mathrm{x}+1046,4$ & 89.24 & 50,94 \\
MST & $\mathrm{Y}^{* *}:-0,0077 \mathrm{x}^{2}+0,7761 \mathrm{x}+8,02$ & 73.99 & 50,40 \\
MSPA & $\mathrm{Y}^{* *}:-0,0027 \mathrm{x}^{2}+0,3018 \mathrm{x}+5,0796$ & 87.05 & 55,89 \\
\hline
\end{tabular}

**: Significativo a $1 \%$ de probabilidade; *: Significativo a $5 \%$ de probabilidade.

Isso, porque o enxofre é um importante constituinte de proteínas (Marschner, 1995) e, a sua ausência, retarda o crescimento vegetal, pois a síntese de importantes proteínas é interrompida (RAIJ, 1991).

\section{CONSIDERAÇÕES FINAIS}

A aplicação de macronutrientes refletiu na maior produção de biomassa das plantas de $P$. angulata. sendo definido a MSPA como o parâmetro que melhor reflete a produção de biomassa, visto que o potencial farmacológico da espécie é explorado na sua parte aérea. As doses de macronutrientes recomendadas para maior incremento da MSPA, são: $\mathrm{N}\left(127,6 \mathrm{mg} . \mathrm{dm}^{-3}\right), \mathrm{P}$ $\left(77,54 \mathrm{mg} \cdot \mathrm{dm}^{-3}\right), \mathrm{K}\left(193,25 \mathrm{mg} \cdot \mathrm{dm}^{-3}\right), \mathrm{Ca}\left(1,27 \mathrm{cmol}_{\mathrm{c}} \cdot \mathrm{dm}^{-3}\right), \mathrm{Mg}\left(0,63 \mathrm{cmol}_{\mathrm{c}} \cdot \mathrm{dm}^{-3}\right)$ e $\mathrm{S}\left(55,89 \mathrm{mg} \cdot \mathrm{dm}^{-}\right.$ $\left.{ }^{3}\right)$.

\section{REFERÊNCIAS BIBLIOGRÁFICAS}

ADAMS, M. et al. Medicinal herbs for the treatment of rheumatic disorders. Journal of Ethnopharmacology, v.121, n.2, p.343-359, 2009.

CORRÊA, M. P. Dicionário das plantas úteis do Brasil e das exóticas cultivadas. Ministério da Agricultura. Instituto Brasileiro de Desenvolvimento Florestal, vol.II, Imprensa Nacional, 1984, $707 \mathrm{p}$.

FERREIRA, D. F. Sisvar: um programa para análises e ensino de estatística. Revista Symposium, v. 6, p. 36-41, 2008.

GOMES, F. T. Nodulação, fixação de nitrogênio e produção de matéria seca de alfafa em resposta a doses de calcário, com diferentes relações cálcio:magnésio. Ciência Rural, Santa Maria, v.32, n.6, p.925-930, 2002

GONÇALVES et al., Crescimento de Dalbergia nigra (Vell.) Allemão ex Benth sob diferentes doses de cálcio, magnésio e enxofre. Revista Árvore, vol. 38, núm. 2, 2014, pp. 251-260 Universidade Federal de Viçosa Viçosa, Brasil.

KERBAUY, G. B. Fisiologia Vegetal. Editora Guanabara Koogan S.A, 2004. MALAVOLTA, E.; VITTI, G. C.; OLIVEIRA, S. A. de. Avaliação do estado nutricional das plantas:

princípios e aplicações. 2. Ed. Piracicaba, 1997. 319 p.

MARSCHNER, H. Mineral nutrition of higher plants. 2.ed. Academic, 1995. 888p

RAIJ, B. Fertilidade do solo e adubação. Piracicaba: Ceres/Potafos, 1991. 343 p.

SHEAR, C. B.; FAUST, M. Nutrition ranger in deciduous tree fruits and nuts. Horticultures

Reviews, n. 2. p. 142-163, 1980.

TURRENT, F. A. Uso de una matriz mixta para la optimización de cinco a ocho factores controlables de la producción, Colégio de Postgraduados, 1979. 65 p.

YASARI, M. A. E. et al. Enhancement of growth and nutrient uptake of rapeseed (Brassica napus L.) by applying mineral nutrients and biofertilizers. Pakistan Journal of Biological Sciences, v.12, p.127-133, 2009. 